\title{
ORÍGENES DE CONTAMINACIÓN DE AGUAS SUBTERRÁNEAS EN UN SECTOR DE LA MARGEN IZQUIERDA DEL RÍO VIRILLA, SAN JOSÉ, COSTA RICA
}

\author{
Gunther Schosinsky, Asdrúbal Vargas \& Jesse Stimson \\ Escuela Centroamericana de Geología \\ Apdo. 214-2060 Universidad de Costa Rica
}

(Recibido 11/9/2000; Aceptado 9/11/2000)

\begin{abstract}
At least four wells located in Rohrmoser, San José had high concentrations of chloride (above $500 \mathrm{ppm}$ ), which were over the drinking water limits set by Acueductos y Acantarillados (Water Supply and Sewage Service). The hydrogeochemical characteristics of groundwater in the urbanized area of the Central Valley were analyzed, which was classified in general as a calcium-magnesium-bicarbonate water which in the majority of cases do not exceed the drinking water limits. Therefore, the anomolous concentrations found in these wells, principally chloride, might be explained by a geothermal source. The hypothesis that the contamination was due to infiltration from the María Aguilar or Torres Rivers was also rejected, due to the fact that these surface waters had low concentrations in chloride. During long pumping tests, the concentrations of chloride and total hardness decreased almost $60 \%$. This was due to the dilution by uncontaminated waters that were drawn to the well from outside of the plume of contamination.
\end{abstract}

\begin{abstract}
RESUMEN: En la vecindad de Rohrmoser, San José, se identificaron al menos cuatro pozos con altas concentraciones de cloruros (sobre los $500 \mathrm{ppm}$ ), que no cumplen con las normas de potabilidad del Instituto Costarricense de Acueductos y Acantarillados. Se analizaron las características hidrogeoquímicas del agua subterránea en la parte urbana del Valle Central, donde el agua de los pozos se clasificó como de tipo bicarbonatada-cálcica-magnésica que en la mayoría de casos cumplen con las normas mencionadas. Por ende, las concentraciones anómalas registradas en estos pozos, principalmente de cloruros, podría deberse a una fuente geotermal. También se descartó la posibilidad de contaminación por infiltración de aguas de los ríos María Aguilar o Torres, por contener bajas concentraciones de cloruro. Durante bombeos prolongados, las concentraciones de cloruros y la dureza total disminuyen en casi un $60 \%$. Esto se debe a la dilución provocada por la afluencia de agua del mismo acuífero, atrayendo agua más allá de la pluma de contaminación.
\end{abstract}

\section{INTRODUCCIÓN}

El área de estudio comprende un sector entre las coordenadas en latitud 211-218 y en longitud 521-527 de la hoja topográfica Abra e incluye las poblaciones de Rohrmoser, Pavas y un sector de Escazú, así como San Juan de Tibás y Lagunilla de Heredia al Norte (Fig. 1).

El objetivo de este trabajo es reunir información sobre las características químicas del agua subterránea en un sector de la margen izquierda del río Virilla. Además en vista de que ciertos pozos del área presentan indicios de contaminación con respecto a los parámetros generales, se pretende analizar el origen de esta contaminación.

El método de trabajo ha consistido en recopilar información y datos químicos del agua subterránea contenidos en los archivos de pozos del Servicio Nacional de Aguas Subterráneas 


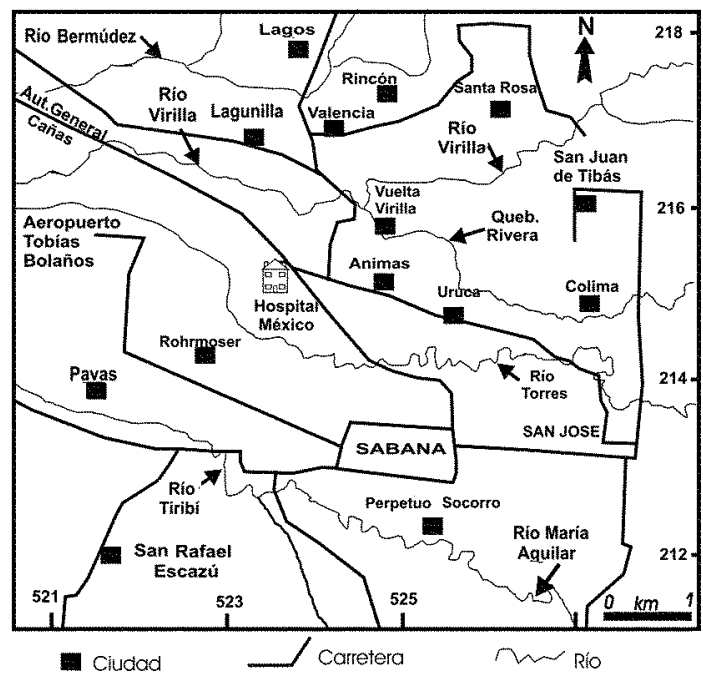

Fig. 1: Localización del área de estudio.

Riego y Avenamiento (SENARA). Además, se obtuvo información por medio de comunicaciones escritas o verbales con los administradores de los pozos. Se midieron temperaturas a diferentes profundidades en el pozo del Instituto Nacional de Seguros. Por otro lado se realizó una campaña de muestreo y análisis de agua superficial en los ríos Torres y María Aguilar en dos puntos seleccionados, con la siguiente ubicación en la cuadrícula Lambert de coordenadas planas: 212,2 norte, 524,9 oeste para el río María Aguilar y 214,4 norte, 523,6 oeste para el río Torres.

La integración de la información recopilada, junto con las características de flujo del agua subterránea, la disposición de los pozos y las condiciones geológicas permitieron describir las características hidrogeoquímicas y proponer una fuente de contaminación responsable de las elevadas concentraciones de ciertas substancias disueltas en el agua subterránea. Pocos estudios sobre hidrogeoquímica se han realizado en este sector, a excepción de un estudio a nivel regional, (Protti et al., 1983). Las fuentes principales de información corresponden con los análisis químicos realizados por AyA y SENAS o por entes privados.

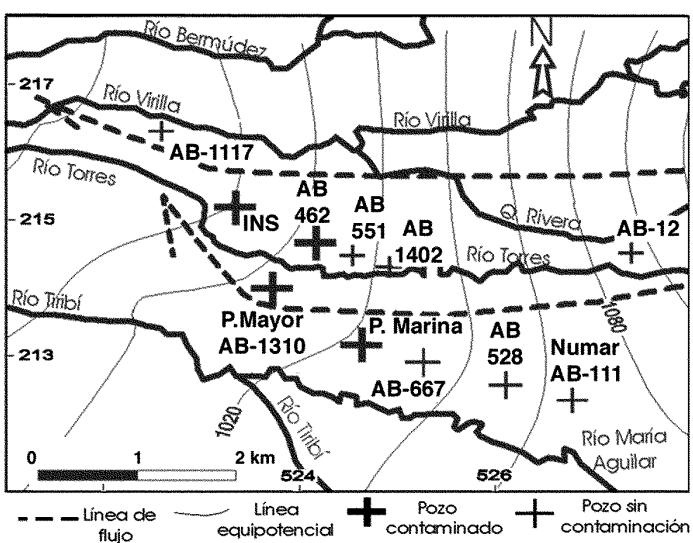

Fig. 2: Pozos con alto contenido de cloruros, fuera de las normas de potabilidad; líneas equipotenciales y líneas de flujo.

\section{INDICIOS DE CONTAMINACIÓN DEL AGUA SUBTERRÁNEA EN LA MARGEN IZQUIERDA DEL RÍO VIRILLA}

Es poco lo que se sabe de la calidad físicoquímica de las aguas subterráneas en la margen izquierda del río Virilla. Sin embargo se tienen evidencias de alto contenido de cloruros, fuera de las normas de potabilidad, en varios pozos ubicados en la zona de la Uruca, Rohrmoser y Sabana oeste. Estos pozos se muestran en la figura 2.

La contaminación de las aguas subterráneas, con altas concentraciones de cloruros, se notó inicialmente en 1971, en el pozo de la Orange Crush en la Uruca (AB-462) con 416 ppm. Posteriormente, se observó el mismo tipo de contaminación en el pozo del Supermercado Plaza Mayor (AB1310) con 640 ppm, en el pozo del Instituto Nacional de Seguros (INS) (532 ppm) en 1999 y por último, en febrero del 2000, el pozo de la Princesa Marina en Sabana oeste, (AB1738) con 3863 ppm, (Fig 2).

Para evaluar más detalladamente la contaminación se recogieron muestras de agua subterráneas, de los tres últimos pozos mencionados, en botellas de plástico de $500 \mathrm{~mL}$, sin presencia 
de burbuja, para determinar parámetros de interés; dentro de ellos, cloruros, hierro total y manganeso, lo que se puede apreciar en el cuadro 1. A la vez, se midió en el campo el pH, la conduc- tividad eléctrica y la temperatura. Es interesante mencionar que estos pozos se encuentran en las márgenes del río Torres, alineados a lo largo de una misma línea de flujo (Fig 2).

\section{Cuadro 1}

Química inorgánica seleccionada de tres pozos de la zona de Uruca

\begin{tabular}{lcccccc}
\hline Pozo & Nombre & Coordenadas & $\begin{array}{c}\text { Cloruros } \\
(\mathrm{ppm})\end{array}$ & $\begin{array}{c}\text { Hierro } \\
\text { Total } \\
(\mathrm{ppm})\end{array}$ & $\begin{array}{c}\text { Maganeso } \\
(\mathrm{ppm})\end{array}$ & $\begin{array}{c}\text { Dureza } \\
\text { Total } \\
(\mathrm{ppm})\end{array}$ \\
\hline AB-1310 & Plaza Mayor & 5523800214000 & 640 & 4,4 & 4,3 & 580 \\
ND & INS & 5233000215200 & 532 & 1,41 & 2,32 & 722 \\
AB-1783 & Princesa Marina & 5242200213080 & 3863 & 0,09 & ND & 2043 \\
\hline
\end{tabular}

$\mathrm{ND}=$ No disponible

En el pozo AB 1783 (coordenadas 213,08 y 524,22), ubicado en el costado oeste de la Sábana, existe un análisis químico más completo. Este pozo, tiene una profundidad de 153 m (rejillas entre 129 y 153 m) que implica que está enrejillado dentro del acuífero Colima Superior. En el cuadro 2, se presenta la química de este pozo.

Con respecto a los demás, este pozo tiene mayor conductividad eléctrica $(14370 \mu \mathrm{S} / \mathrm{cm})$ y mayor concentración de cloruros (3 $863 \mathrm{mg} / \mathrm{l}$ ).

$\mathrm{Al}$ considerar los elevados valores de conductividad eléctrica, dureza total y las altas concentraciones de manganeso así como de sólidos totales en los pozos contaminados, lo que se observa en los análisis del pozo AB 1783 (cuadro 2), se considera que la dureza total y manganeso, podrían provenir de las formaciones geológicas, mientras que la elevada contaminación por cloruros puede ser de origen antropogénico.

\section{ESTUDIO DE LA CALIDAD DE LAS AGUAS SUBTERRÁNEAS}

En marzo del 2000, se recopilaron datos hidrogeoquímicos existentes en el Servicio Nacional de Aguas Subterráneas, Riego y Avenamiento (SENARA). Se realizó una revisión detallada de todos los archivos de pozos ubicados en
Cuadro 2

Química inorgánica del agua del pozo AB 1783

\begin{tabular}{|c|c|c|}
\hline Determinación & Resultado & \\
\hline $\mathrm{pH}$ & 6,03 & \\
\hline Turbidez & 88 & UT \\
\hline Color & 75 & $\mathrm{UC}$ \\
\hline Conductividad eléctrica & 14370 & $\mu \mathrm{S} / \mathrm{cm}$ \\
\hline Sólidos totales & 9760 & ppm \\
\hline Sales disueltas & 8500 & ppm \\
\hline Sólidos suspendidos & 1260 & ppm \\
\hline Alcalinidad parcial (F.F.) & $<1$ & ppm \\
\hline Alcalinidad total (R.M.) & 1459 & ppm \\
\hline Dureza total (como $\mathrm{CaCO}_{3}$ ) & 2043 & ppm \\
\hline Dureza carbonatos (como $\mathrm{CaCO}_{3}$ ) & 1459 & ppm \\
\hline Dureza no carbonatos $\left(\right.$ como $\left.\mathrm{CaCO}_{3}\right)$ & 684 & ppm \\
\hline Calcio $\left(\mathrm{Ca}^{2+}\right)$ & 363 & ppm \\
\hline Magnesio $\left(\mathrm{Mg}^{2+}\right)$ & 276 & ppm \\
\hline Cloruros $\left(\mathrm{Cl}^{-}\right)$ & 3863 & ppm \\
\hline Sulfatos $\left(\mathrm{SO}^{2-}\right)$ & 172 & ppm \\
\hline Sílice $\left(\mathrm{SiO}_{2}\right)$ & 45 & ppm \\
\hline Hierro total $\left(\mathrm{Fe}_{\text {total }}\right)$ & 0,090 & ppm \\
\hline
\end{tabular}

el sector de estudio, ubicado en la hoja cartográfica ABRA del Instituto Geográfico Nacional de Cosa Rica, que coincide en gran parte con el área metropolitana de San José y sus comunidades aledañas, incluyendo La Uruca, Tibás, Guadalupe, San Pedro, Curridabat, Zapote y Desamparados.

Es interesante notar que entre 1970 y 1975, se llevó un control estricto de los pozos 
perforados incluyendo análisis químico de sus aguas Sin embargo, después de 1975, no se continuó con esa exigencia por parte del SENARA, por ende, existen muy pocos datos sobre la química de las aguas subterráneas, después de ese año.

Los datos recopilados de 58 muestras de 32 pozos, indican que las aguas subterráneas son de buena calidad, son aguas cálcica magnésicas bicarbonatadas diluidas que no llegan a sobrepasar las normas de potabilidad de agua.

Por ejemplo, la conductividad eléctrica de los pozos examinados, (no incluyendo los pozos contaminados), varía entre 180 y 360 $\mu \mathrm{S} / \mathrm{cm}$, con un promedio de $261 \mu \mathrm{S} / \mathrm{cm}$. La poca variabilidad de estos datos (desviación estándar, $\sigma=47$ ), indica que el valor medido en el pozo de Princesa Marina de la Sabana (14 $370 \mu \mathrm{S} / \mathrm{cm}$ ), proviene de una fuente que no es típica en las aguas subterráneas en el área de estudio.

También las concentraciones de sólidos disueltos totales en el pozo Princesa Marina (9760 mg/l) están muy por encima de las concentraciones típicas, que varían entre 483 y $69 \mathrm{mg} / \mathrm{l}$ (excepto el pozo AB-117, cine Rex, con 1327 $\mathrm{mg} / \mathrm{l})$, tienen una concentración promedio de 192 mg/l, y una concentración mediana de $180 \mathrm{mg} / \mathrm{l}$.

La concentración de cloruros, excluyendo los pozos contaminados, varía entre 0,7 y 50 $\mathrm{mg} / \mathrm{l}$, con una concentración promedio de 12,0 $\mathrm{mg} / \mathrm{l}$ y una concentración mediana de $8 \mathrm{mg} / \mathrm{l}$, que no se comparan con las concentraciones mayores a $400 \mathrm{mg} / \mathrm{l}$ que se midieron en los pozos de Uruca, Sabana y Rohrmoser.

La dureza total, también es más alta en los pozos contaminados (de 256 a $2043 \mathrm{mg} / \mathrm{l}$ ) comparada con los valores medidos en el área de estudio, que varía entre 13 y 242,5 mg/l, con una concentración promedio de $104 \mathrm{mg} / \mathrm{l}$ y una concentración mediana de $94 \mathrm{mg} / \mathrm{l}$.

El hierro total de los pozos contaminados con cloruros, tienen concentraciones entre 1 y 4 $\mathrm{mg} / \mathrm{l}$ (Cuadro 1), que es comparable con algunas de las aguas del área de estudio y zonas cercanas, donde las concentraciones mayores a $1 \mathrm{mg} / \mathrm{l}$ existen en 15 de 58 muestras ( $26 \%$ del total). Además, el pozo de Princesa Marina, con muy alto contenido de cloruros, se registró una concentración de hierro total muy baja $(0,09 \mathrm{mg} / \mathrm{l})$.

Es interesante notar, que en diciembre de 1971, se registraron concentraciones altas en dos muestreos realizados en el pozo AB-462, de la Orange Crush, con valores de 416 y $258 \mathrm{mg} / \mathrm{l}$ de cloruros. Eso indica, que la contaminación ha existido en la zona desde hace 30 años.

\section{Temperaturas medidas a diferentes profundidades en el pozo del INS}

El 21 de mayo de 1999, se realizaron medidas de temperatura y conductividad eléctrica en el pozo del INS, utilizando una sonda que permite ambas lectura a diferentes profundidades (Cuadro 3).

La temperatura del pozo del INS se encuentra sobre la temperatura promedio de las aguas subterráneas del área de estudio. De los archivos del SENARA, se recopilaron 34 muestras de 17 pozos ubicados en el área de estudio y sus alrededores (Cuadro 4), indicando que la temperatura varía entre 21 y $26^{\circ} \mathrm{C}$, con una temperatura promedio de $23,8^{\circ} \mathrm{C}$. La figura 3 , muestra una correlación entre la profundidad del pozo y la temperatura del agua, donde se puede observar que los pozos contaminados son los que muestran las más altas temperaturas.

\section{Cuadro 3}

Temperatura y conductividad eléctrica a diferentes profundidades en el pozo de INS

\begin{tabular}{|c|c|c|c|c|c|c|c|c|c|c|c|}
\hline Profundidad (m) & 78,4 & 80,4 & 84,4 & 86,4 & 88,4 & 90,4 & 92,4 & 94,4 & 96,4 & 98,4 & 100,4 \\
\hline Temperatura $\left({ }^{\circ} \mathrm{C}\right)$ & 26,2 & 26,2 & 26,2 & 26,3 & 26,4 & 26,5 & 26,5 & 26,6 & 26,7 & 26,7 & 27,0 \\
\hline Conductividad $(\mu \mathrm{S} / \mathrm{cm})$ & 1180 & 1462 & 1439 & 1432 & 1429 & 1427 & 1425 & 1425 & 1423 & 1424 & 1720 \\
\hline
\end{tabular}

Nivel Est. $=78,40 \mathrm{~m}$ 
Cuadro 4

Temperatura a diferentes profundidades y fechas en pozos del área de estudio

\begin{tabular}{lccccccccccc}
\hline Pozo & Long. & Lat. & $\begin{array}{c}\text { Fecha de } \\
\text { medida }\end{array}$ & $\begin{array}{c}\mathrm{T} \\
\left({ }^{\circ} \mathrm{C}\right)\end{array}$ & $\begin{array}{c}\text { Prof } \\
(\mathrm{m})\end{array}$ & Pozo & Long. & Lat. & $\begin{array}{c}\text { Fecha de } \\
\text { medida }\end{array}$ & $\begin{array}{c}\mathrm{T} \\
\left({ }^{\circ} \mathrm{C}\right)\end{array}$ & $\begin{array}{c}\text { Prof. } \\
(\mathrm{m})\end{array}$ \\
\hline AB-206 & 530,76 & 210,6 & $17-11-71$ & 26 & 124 & AB-52 & 528,44 & 213,24 & $3-05-72$ & 24 & 35 \\
AB-198 & 527,4 & 210,56 & $7-05-71$ & 25,5 & 35 & AB-111 & 526,61 & 212,16 & $26-04-73$ & 24 & 55 \\
AB-12 & 527,6 & 214,5 & $5-05-72$ & 25 & 20 & AB-182 & 530,5 & 212,04 & $26-04-73$ & 24 & 152 \\
AB-182 & 530,5 & 212,04 & $15-11-71$ & 25 & 152 & AB-192 & 531,21 & 211,02 & $26-04-73$ & 24 & 61 \\
AB-192 & 531,21 & 211,02 & $17-11-71$ & 25 & 61 & AB-206 & 530,76 & 210,6 & $26-04-73$ & 24 & 124 \\
AB-224 & 529,34 & 209,12 & $5-05-72$ & 25 & & AB-228 & 531,25 & 209,34 & $5-12-78$ & 24 & 68 \\
AB-52 & 528,44 & 213,24 & $26-04-73$ & 24,5 & 35 & AB-192 & 531,21 & 211,02 & $6-05-71$ & 23,5 & 61 \\
AB-4 & 526,4 & 215,10 & $18-11-73$ & 24 & & AB-91 & 530,6 & 213 & $2-05-71$ & 23 & 9 \\
AB-183 & 530,4 & 212,10 & $6-$-May-71 & 24 & 152 & AB-182 & 530,5 & 212,04 & $6-05-71$ & 23 & 152 \\
AB-190 & 531,17 & 211,22 & $29-11-71$ & 24 & 72 & AB-59 & 529,23 & 213,81 & $18-11-71$ & 23 & 61 \\
AB-190 & 531,17 & 211,22 & $4-05-72$ & 24 & 72 & AB-52 & 528,44 & 213,24 & $15-11-71$ & 23 & 35 \\
AB-182 & 530,5 & 212,04 & $4-05-72$ & 24 & 152 & AB-211 & 532,14 & 210,26 & $5-05-72$ & 23 & 73 \\
AB-192 & 531,21 & 211,02 & $4-05-72$ & 24 & 61 & AB-12 & 527,6 & 214,5 & $23-11-71$ & 22 & 20 \\
AB-194 & 532,3 & 211,05 & $3-05-72$ & 24 & 225 & AB-528 & 526 & 213,1 & $07-11-73$ & 22 & 37 \\
AB-206 & 530,76 & 210,6 & $4-05-72$ & 24 & 124 & AB-111 & 526,61 & 212,16 & $23-11-71$ & 22 & 55 \\
AB-110 & 526,61 & 212,4 & $02-05-72$ & 24 & 55 & AB-59 & 529,23 & 213,81 & $2-05-73$ & 22 & 61 \\
AB-111 & 526,61 & 212,16 & $02-05-72$ & 24 & 55 & AB-609 & 531,4 & 211,4 & $29-07-75$ & 21 & 70 \\
\hline
\end{tabular}

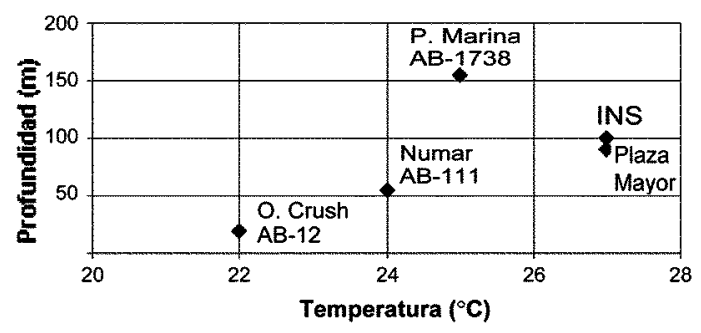

Fig. 3: Temperatura vs. profundidad en algunos pozos del área de estudio.

El mismo día en que se midió la temperatura, se realizó una medida de nitratos en el pozo, a una profundidad de 80 metros, obteniendo un resultado de $6 \mathrm{ppm}$ lo cual es muy bajo.

\section{Otras propiedades del agua en el pozo del INS}

Cuando se bombeó por primera vez este pozo el agua mostraba un $\mathrm{pH}$ de 7,31, cloruros de 532 ppm, sodio 626 ppm, potasio 140 ppm y $\mathrm{CO}_{2}$ disuelto superior a $450 \mathrm{ppm}$. Además, el agua levemente tibia, con un fuerte sabor de agua carbonatada. Cuando se agitó el agua dentro de una botella, se formaron pequeñas burbujas de gas, que al destapar la botella, salió a presión. Esas burbujas y el sabor del agua se le atribuyo a la presencia de $\mathrm{CO}_{2}$.

Durante un bombeo prolongado de 10 días, los cloruros y dureza total disminuyeron en un 60 por ciento. Esto se debe a una disminución de los cloruros por disolución, debido a que el cono de influencia durante el bombeo, atrae agua del mismo acuífero más allá de la pluma de contaminación.

\section{POSIBLE ORIGEN DE LA CONTAMINACIÓN}

La contaminación detectada puede tener varios orígenes: producto de un botadero de basura, un pozo industrial en donde se inyecten contaminantes, una fuente natural de agua geotermal, infiltración de agua desde el río Torres o de aguas marinas fósiles. La contaminación se ha detectado en cuatro pozos en un área aproximada de seis kilómetros cuadrados; sin embargo un pozo muy cercano al de la empresa Orange Crush (AB-551), no mostró contaminación de acuerdo 
con los análisis químicos de aguas subterráneas realizados en 1975.

\section{Lixiviados desde un botadero de basura}

A una distancia de un $1500 \mathrm{~m}$ y en dirección Este de los pozos contaminados (INS, Plaza Mayor) se encuentra un antiguo basurero o crematorio. Este sitio fue utilizado para disponer desechos durante los años 70; sin embargo, actualmente no se encuentra en funcionamiento. Analizando las líneas de flujo del agua subterránea se nota que la dirección de flujo es NE, mientras que los pozos se encuentran al NW, por lo tanto se descarta la posibilidad que lixiviados desprendidos desde este botadero puedan alcanzar los pozos.

\section{Origen industrial}

Los pozos contaminados se disponen a lo largo de una zona más o menos rectangular y de orientación NW. Sin embargo, no se ha logrado relacionar la contaminación de los pozos con alguna industria presente en la zona, aunque no puede descartarse completamente esta posibilidad.

\section{Aguas marinas fósiles}

Varios análisis químicos muestran una elevada concentración en sulfatos, cloruros y bicarbonato, lo cual es similar a las concentraciones de aguas marinas. Además, se debe considerar la presencia en el área de estudio de formaciones rocosas de tipo sedimentario marino profundo. Sin embargo, esta es una hipótesis que pierde validez, al considerar que existe un flujo de agua subterránea, en estas formaciones fracturadas y permeables que tendería a diluir los solutos presentes en el agua.

\section{Aguas Termales}

La posibilidad de que la fuente de contaminación tuviese su origen en aguas marinas fósiles, lixiviados de un basurero o de origen industrial, pierde validez de acuerdo con lo mencionado anteriormente. Sin embargo, quedan dos hipótesis por resolver: si las aguas provienen de los ríos Torres o María Aguilar o tienen influencia de una fuente termal.
Según los mapas geológicos no existen fallas evidentes ni manantiales termales en la zona. Existe información de que luego del terremoto de Puriscal en 1988, varias casas de habitación de Rohrmoser quedaron dañadas a lo largo de una franja más o menos lineal, probablemente a lo largo de una falla; sin embargo, sismólogos de la Red Sismológica Nacional han descartado esta posibilidad y más bien señalan la causa de los daños al tipo de suelo del área afectada.

Una de las características de las aguas termales es que en la mayoría de ellas se encuentra un alto contenido de cloruro y de sodio. Además, contienen altas proporciones de bicarbonatos, cuando el $\mathrm{pH}$ se encuentra entre 7 y 8 . Estas aguas muestran alta temperatura que disminuyen a medida que el agua transita a lo largo del acuífero.

El pH de 7,3, los altos contenidos de cloruros, bicarbonatos, la elevada temperatura del agua en el pozo del INS con respecto a la temperatura del área de estudio, hacen sospechar que las altas concentraciones de los elementos mencionados, se deben a un origen termal. Clasificando estas aguas dentro del sistema geotérmico (por sus aniones y $\mathrm{pH}$ ) resultan ser cloruro-bicarbonatadas.

\section{Ríos de tipo influente}

La contaminación se ha detectado en un área muy cercana al río Torres, donde seis pozos presentan contaminación. Sin embargo un pozo muy cercano al de la Orange Crush, el AB551, no mostró contaminación de acuerdo con los análisis químicos de aguas subterráneas realizadas en 1975.

Otro aspecto interesante es el hecho de que la contaminación se detectó en el año de 1972 y se pensaría que el río Torres no presentaría contaminación suficiente para esa época, sin embargo la información recolectada en los periódicos nos indica que ya para esa época existía una dramática contaminación ambiental. En el diario la República del 14 de octubre de 1975 se indica:

\footnotetext{
“...La contaminación alcanza también a los ríos, que son el origen de la contaminación de las aguas expresó el ingeniero Edison Rivera Castaing. Los ríos son generalmente el depósito de los desechos
} 
sólidos originados en basuras domésticas e industriales. Es común que la basura se tire a un río, no solo eso sino que además se arrojan autos viejos, ramas, serrín de los aserraderos. La más afectada por este tipo de contaminación es el Area Metropolitana dónde en la décima parte del trerritorio nacional se agrupan un millón de personas “.

En el sector que comprende a los pozos INS, Plaza Mayor y AB-462 el río Torres fluye sobre ignimbritas, lo cual podría favorecer la contaminación; sin embargo, en el sector donde se encuentran los pozos AB-528 y AB-12 el río Torres fluye sobre lahares los cuales podría funcionar como una capa filtro de los contaminantes.

\section{CONCENTRACIONES DE INDICADORES DE CONTAMINACIÓN EN LOS RÍOS TORRES Y MARÍA AGUILAR}

En la figura 4, se puede observar que los ríos Torres y María Aguilar recargan al acuífero, o sea que son influentes en un tramo del río. Para determinar si la contaminación observada en los po- zos de Uruca, Rohrmoser y Sabana, proviene de los río Torres y María Aquilar, se tomaron muestras de estos dos ríos a diferentes horas del día y en diferentes días de la semana, dado que las descargas al río son muy variables a través del tiempo. Esta información se presenta en los cuadros 5 y 6 .

El muestreo del río María Aguilar se hizo en un punto a $200 \mathrm{~m}$ al norte del Centro Comercial Plaza Mayor. El muestreo del río Torres se realizó en un punto $700 \mathrm{~m}$ al sur del Parque la Sábana. Debido a las altas concentraciones de materia suspendida en esas aguas contaminadas, las muestras de río se filtraron mediante un filtro de $0.45 \mu \mathrm{m}$ antes de ser analizadas en el laboratorio.

Se puede observar que los cloruros se mantienen aproximadamente dentro de 45 y 30 ppm, tanto en el río Torres como en el María Aguilar, por lo que se deduce que las elevadas concentraciones de los cloruros en los pozos de la Uruca, Rohrmoser y Sabana no provienen de los ríos mencionados.

Los valores bajos de cloruros y conductividad eléctrica, del día 23 de junio del 2000, en ambos ríos, se debe a que en este día se encontraba lloviendo. Sin embargo, el mismo día, las concentraciones de nitrato eran las más altas, por el lavado de fuentes de nitrato hacia los ríos. Por

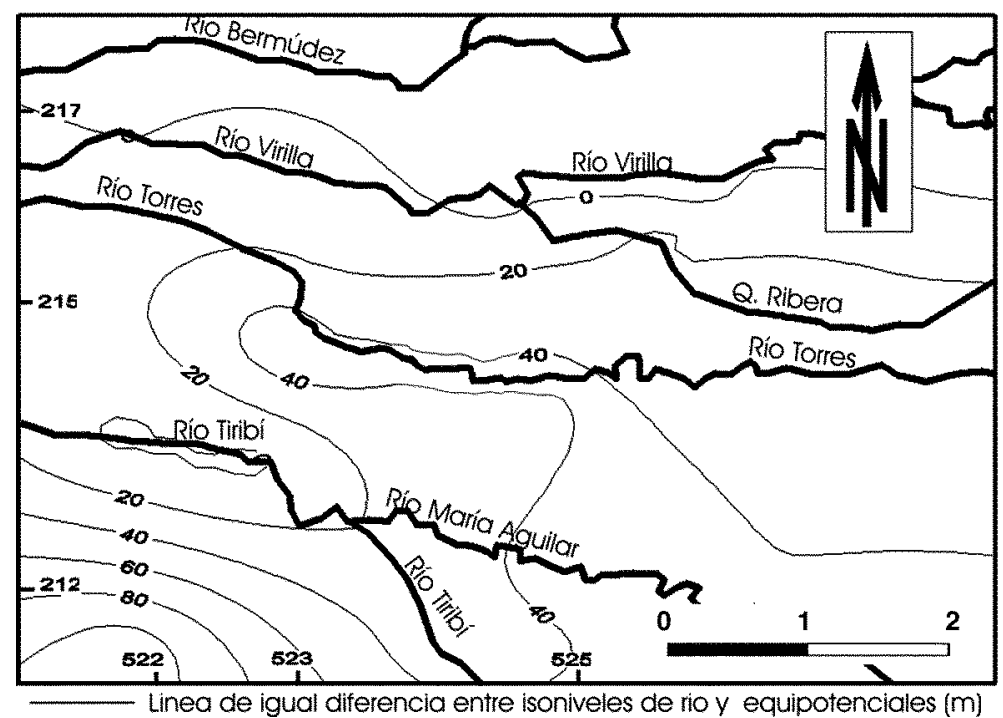

Fig. 4: Condiciones de influencia o efluencia de los ríos Torres y María Aguilar sobre los acuíferos indicando los valores positivos influencia del río sobre el acuífero. 
Cuadro 5

Química inorgánica del río Torres

\begin{tabular}{|c|c|c|c|c|c|c|}
\hline FECHA & HORA & $\mathrm{PH}$ & $\begin{array}{l}\mathrm{Cl}- \\
\mathrm{ppm}\end{array}$ & $\begin{array}{c}\text { Conductividad } \\
\mu \mathrm{S} / \mathrm{cm}\end{array}$ & $\begin{array}{l}\text { SO4-2 } \\
\text { ppm }\end{array}$ & $\begin{array}{l}\text { NO3- } \\
\text { ppm }\end{array}$ \\
\hline 25 Ago 1999 & $10: 00 \mathrm{am}$ & ND & 40 & ND & ND & 8 \\
\hline 7 Abr 2000 & 11:00 am & 7,32 & 44 & 443 & 31,1 & 118 \\
\hline 15 May 2000 & $7: 30 \mathrm{am}$ & 7,15 & 36 & 443 & 31,1 & $<0,5$ \\
\hline 15 May 2000 & $12: 00 \mathrm{~m}$ & 7,60 & 32 & 427 & 32,1 & 1 \\
\hline 23 Jun 2000 & 8:00 am & 7,10 & 28 & 321 & 28,6 & 87 \\
\hline 23 Jun 2000 & $1: 30 \mathrm{pm}$ & 7,39 & 30 & 319 & 29,4 & 65 \\
\hline
\end{tabular}

$\mathrm{ND}=$ no disponible

Cuadro 6

Química inorgánica del río María Aguilar

\begin{tabular}{lcccccc}
\hline FECHA & HORA & PH & $\begin{array}{c}\mathrm{Cl}- \\
\mathrm{ppm}\end{array}$ & $\begin{array}{c}\text { Conductividad } \\
\mu \text { S/cm }\end{array}$ & $\begin{array}{c}\text { SO4-2 } \\
\text { ppm }\end{array}$ & $\begin{array}{c}\text { NO3- } \\
\text { ppm }\end{array}$ \\
\hline 7 Abr 2000 & $11: 30 \mathrm{am}$ & 7,21 & 42 & 441 & 37,8 & $<0,5$ \\
15 May 2000 & $8: 00 \mathrm{am}$ & 6,95 & 36 & 326 & 34,1 & 56 \\
15 May 2000 & $12: 30 \mathrm{~m}$ & 7,30 & 32 & 323 & 32,1 & 67 \\
23 Jun 2000 & $8: 30 \mathrm{am}$ & 7,25 & 30 & 297 & 27,0 & 108 \\
23 Jun 2000 & $2: 00 \mathrm{pm}$ & 7,35 & 34 & 280 & 27,0 & 89 \\
\hline
\end{tabular}

$\mathrm{ND}=$ no disponible

ende, se puede notar que la conductividad eléctrica generalmente aumenta con el incremento de la concentración de cloruros y con la disminución de la concentración de nitratos.

\section{RESULTADOS Y CONCLUSIONES}

A partir de la información obtenida, se tiene como probable que el origen de la contaminación del agua subterránea de los pozos identificados como INS, Plaza Mayor, Orange Crush y Princesa Marina, sea de origen termal.

De todas las hipótesis posibles que han sido analizadas para determinar el origen de los altos cloruros, conductividad eléctrica, sólidos disueltos totales y dureza total, ha quedado una sola sin perder validez; esta hipótesis es que las aguas son de origen termal. Esta se refuerza, por la temperatura superior que mostró el pozo del INS. Sin embargo, no se descarta la posibilidad, que una industria este inyectando agua de desecho al acuífero. Para descartar esta posibilidad se necesita un estudio de isótopos; el cual vendría a desechar cualquier otra hipótesis ya que los isótopos del oxígeno y el deuterio se desplazarian hacia la derecha en la curva de agua de origen meteórico.

\section{REFERENCIA}

PROTTI, E., SIU, D., ZARATE, E., RAMÍREZ, P., BERGOEING, J., BRENES, L., ECHANDI, E. \& MONTERO,W., 1983: Sistema fluvial de Tárcoles, Costa Rica: estudios preliminares sobre geomorfometría y geofactores básicos, contribución a la geografía general de Costa Rica. - 90 págs + mapas, IGN-CONICIT, San José. 